levels of workforce pressure (Nursing Standard/Marie Curie survey, 2018) This work also highlights the importance of considering impacts of volunteer services beyond immediate beneficiaries.

\section{P-198 REDUCING AVOIDABLE READMISSIONS FOR PEOPLE WITH COMPLEX NEEDS ASSOCIATED WITH FRAILTY}

Kerry Bareham. St Barnabas Lincolnshire Hospice, Lincoln, Lincolnshire

\subsection{6/bmjspcare-2018-hospiceabs.223}

Older people are the most frequent users of services and when admitted to hospitals are at greater risk of complications leading to longer lengths of stay and readmission rates. The current design of secondary care services fail to identify the complex underlying needs of older people associated with frailty, including palliative care needs. This results in poor discharge planning and subsequent avoidable future admissions. However, for those people who are admitted to community palliative care services their risk of a readmission to hospital within 28 days is halved.

This presentation aims to share the findings of a service transformation project implemented by the author as part of their MSc dissertation. This was achieved with the application of an Experienced Based Design approach with other stakeholders, along with the principles of Six Sigma to understand the root cause, and was supported by a systematic review of the literature. Which informed the case for the role of an Anticipatory Care Nurse based in A and E of an acute trust to educate front door clinicians how to identify people in their last year of life and develop person centred care planning.

An action research and rapid appraisal technique was applied through the use of the PDSA cycle was implemented to evaluate the service transformation project.

A $45 \%$ increase in the number of referrals to the Specialist Palliative Care Team and subsequent increase in referrals to community palliative care services was demonstrated. This was achieved by improved identification of need by front-line clinicians, resulting in widening access to hospice and palliative and end of life care and subsequent person- centred care planning and well-being. Thus, offering a solution to the STP aspiration to reduce avoidable admissions to hospital by $30 \%$. Lincoln University are keen to support further research through expansion of this project.

\section{P-199 CAN WE ENABLE MORE PEOPLE TO LIVE WELL AND DIE IN A PLACE AND MANNER OF THEIR CHOOSING?}

Keri Thomas. The National GSF Centre for End of Life Care, London, UK

\subsection{6/bmjspcare-2018-hospiceabs.224}

Aims A third of hospital patients are in their last year of life, yet approximately $12 \%$ are seen by specialist palliative care leaving generalists to care for $88 \%$ of patients. Generalists need to be enabled to provide good palliative care for their patients. The transition from reactive to proactive care by earlier recognition and assessment of personal needs ensures patients receive better coordinated care in a place and manner of their choosing.

Gold Standards Framework (GSF) is a quality improvement programme delivering proactive person-centred care for people in the last years of life in all settings. We report on the introduction and impact of the GSF Acute Hospitals training Programme, with findings from the 12 whole hospital programmes and accredited wards.

Method The structured two year GSF Hospital training programme includes training, tools, support and evaluation at organisational, patient and staff level, including measures of progress and attainment. The programme is delivered to the whole hospital (commissioned) or individual wards (open). GSF encourages teams to identify patients earlier (aiming for the 30\% figure where appropriate), assess their needs (offering all initial advance care planning discussions) and proactively planning care aligned to preferences, enabling more to die at home if that is their wish.

Results Evaluations indicate significant progress in several key areas in many hospitals and a 'culture change' on the wards. GSF Accredited wards show embedded good practice including earlier identification (average 34\%), more proactive needsbased approach, more offered advance care planning discussions (76\%-87\%), better communication with GPs, reduced hospitalisation and improved more appropriate referrals to palliative care specialists.

Conclusions Findings from the GSF trained hospitals and accredited wards show significant changes, with proactive, better coordinated, person-centred care. A key factor is earlier recognition and accredited wards are able to demonstrate they are identifying $30 \%$ of patients.

\section{P-200 QUALITY IMPROVEMENT IN ACTION - IMPROVING CARE FOR PATIENTS ADMITTED TO HOSPITAL IN AN EMERGENCY}

Julia Grant, Karen Murray, Valerie Olivant, Lizzie Hyde, Kate Russ. University Hospitals Leicester, Leicester, UK

\subsection{6/bmjspcare-2018-hospiceabs.225}

Background University Hospitals Leicester (UHL) joined a quality improvement collaborative (ECLHIP) to improve care for patients who maybe in their last three months of life who attend or are admitted to hospital in an emergency. Hospitals are an important provider of care for this group. UHL is one of the largest Trusts in the English NHS, caring for over 2000 patients in their last days of life each year. An emergency admission may indicate underlying clinical decline. Quality of care varies and there may be unwanted inpatient stays and/or treatments contributing to overall experience.

Method The collaborative begins with diagnosis (activity data, qualitative casefile review, patient experience walkthrough, review of reports and plans). A driver diagram illustrates the 'theory of change' based on findings to plan improvements. The model for improvement provides the framework for engaged frontline clinicians to conduct tests of change. The approach includes informative evaluation, patient and relative experience and planning for sustainability.

Results 62\% ( $n=1991)$ emergency, adult patients who died in UHL had an outpatient appointment, emergency admission, attendance and/or planned admission in the 90 days preceding their final admission. Casefile review: 7/10 patients had likely missed opportunities to plan ahead. During the final admission, uncertain recovery and/or dying was recognised but this was often late and/or did not translate into desired action. A survey of A and E staff indicated gaps in knowledge on how to access SPC (24\% confident) and communication around 